\title{
An inequality between the James and James type constants in Banach spaces
}

\author{
by \\ Fenghui Wang (Luoyang) and Changsen Yang (Xinxiang)
}

\begin{abstract}
We consider the James and Schäffer type constants recently introduced by Takahashi. We prove an equality between James (resp. Schäffer) type constants and the modulus of convexity (resp. smoothness). By using these equalities, we obtain some estimates for the new constants in terms of the James constant. As a result, we improve an inequality between the Zbăganu and James constants.
\end{abstract}

1. Introduction. Recently, the problem of finding the relation between the James constant $J(X)$ and the von Neumann-Jordan constant $C_{\mathrm{NJ}}(X)$ has been investigated by several authors. This problem was originally studied by Kato, Maligranda and Takahashi [16] who proved

$$
C_{\mathrm{NJ}}(X) \leq \frac{[J(X)]^{2}}{1+[J(X)-1]^{2}} .
$$

Since then much effort has gone into improving this inequality; see e.g., [2, 17 22, 24, 25. There are several methods for estimating $C_{\mathrm{NJ}}(X)$. Among them we mention one due to Alonso, Martín and Papini [2], which mainly relies on the inequality

$$
C_{\mathrm{NJ}}^{\prime}(X) \leq J(X),
$$

where $C_{\mathrm{NJ}}^{\prime}(X)$ is a constant introduced by Gao [12]. In [21] Wang improved $(1.2)$ as

$$
C_{\mathrm{NJ}}^{\prime}(X) \leq 1+\frac{4(J(X)-1)^{2}}{J^{2}(X)} .
$$

In addition, Wang and Pang [22] obtained a similar inequality:

$$
A_{2}(X) \leq 1+\sqrt{J(X)-1},
$$

2010 Mathematics Subject Classification: Primary 46B20.

Key words and phrases: modulus of convexity, modulus of smoothness, James constant, Schäffer constant, von Neumann-Jordan constant, Zbăganu constant. 
where $A_{2}(X)$ is a constant introduced by Baronti, Casini and Papini [6]. Later, inequality (1.4) was improved independently by Takahashi and Kato [20] and Wang [21] as

$$
A_{2}(X) \leq \frac{3 J(X)-2}{J(X)} .
$$

This inequality enabled them to improve 1.1 as

$$
C_{\mathrm{NJ}}(X) \leq J(X)
$$

which was also obtained by Yang and $\mathrm{Li}[25]$.

It is readily seen that inequalities $1.2-1.5$ play an important role in estimating the von Neumann-Jordan constant. We note that both $A_{2}(X)$ and $C_{\mathrm{NJ}}^{\prime}(X)$ fall into the class of James type constants, recently introduced by Takahashi [19]. The aim of this paper is to present a general method for estimating James type constants. Moreover we get an inequality between the James and Schäffer type constants from an equality for the modulus of smoothness. As an application, we strengthen an inequality between the Zbăganu and James constants.

2. Preliminaries and notation. Throughout this paper, $B_{X}$ and $S_{X}$ respectively denote the unit ball and the unit sphere of a Banach space $X$. The von Neumann-Jordan constant, introduced by Clarkson [9], is defined as

$$
C_{\mathrm{NJ}}(X)=\sup \left\{\frac{\|x+y\|^{2}+\|x-y\|^{2}}{2\left(\|x\|^{2}+\|y\|^{2}\right)}: x \in S_{X}, y \in B_{X}\right\} .
$$

A Banach space $X$ is called uniformly nonsquare ((UNS) for short), in the sense of James, if there exists a positive number $\delta<2$ such that

$$
\min (\|x-y\|,\|x+y\|) \leq \delta
$$

for all $x, y \in S_{X}$. The James constant

$$
J(X)=\sup \left\{\min (\|x+y\|,\|x-y\|): x, y \in S_{X}\right\}
$$

is introduced to characterize this concept: obviously $X$ is (UNS) in the sense of James if and only if $J(X)<2$. Now let us turn to another definition of uniform nonsquareness. A Banach space $X$ is called uniformly nonsquare in the sense of Schäffer if there exists a $\lambda>1$ such that

$$
\max (\|x-y\|,\|x+y\|) \geq \lambda
$$

for all $x, y \in S_{X}$. The Schäffer constant, defined by

$$
S(X)=\inf \left\{\max (\|x-y\|,\|x+y\|): x, y \in S_{X}\right\},
$$

is introduced to characterize this concept: obviously $X$ is uniformly nonsquare in the sense of Schäffer if and only if $S(X)>1$. From the equality 
$J(X) S(X)=2$ (see [16]), we know that these two definitions of (UNS) are equivalent. For more information on these constants, we refer to [13, 14, 16].

Recall the generalized mean defined by

$$
\mathcal{M}_{t}(a, b):=\left(\frac{a^{t}+b^{t}}{2}\right)^{1 / t},
$$

where $a$ and $b$ are two positive real numbers. It is well known that $\mathcal{M}_{t}(a, b)$ is nondecreasing and

$$
\begin{aligned}
& \mathcal{M}_{-\infty}(a, b):=\lim _{t \rightarrow-\infty} \mathcal{M}_{t}(a, b)=\min (a, b), \\
& \mathcal{M}_{+\infty}(a, b):=\lim _{t \rightarrow+\infty} \mathcal{M}_{t}(a, b)=\max (a, b),
\end{aligned}
$$

and $\mathcal{M}_{0}(a, b)=\lim _{t \rightarrow 0} \mathcal{M}_{t}(a, b)=\sqrt{a b}$. By using the generalized mean, we now recall two classes of geometric constants, which include the James and Schäffer constants, respectively.

Definition 2.1 (Takahashi [19]). (1) For $\tau \geq 0$ and $t \in[-\infty,+\infty$ ), a James type constant is defined by

$$
J_{X, t}(\tau)=\sup \left\{\mathcal{M}_{t}(\|x-\tau y\|,\|x+\tau y\|): x, y \in S_{X}\right\} .
$$

(2) For $\tau \geq 0$ and $1<t \leq+\infty$, a Schäffer type constant is defined by

$$
S_{X, t}(\tau)=\inf \left\{\mathcal{M}_{t}(\|x-\tau y\|,\|x+\tau y\|): x, y \in S_{X}\right\} .
$$

Obviously $J_{X, t}(\tau)$ includes some known constants, such as Alonso-Llorens-Fuster's constant $T(X)$ [1], Baronti-Casini-Papini's constant $A_{2}(X)$ [6], Gao's constant $C_{\mathrm{NJ}}^{\prime}(X)$ [12] and Yang-Wang's modulus $\gamma_{X}(t)$ [26]. Also $S_{X, t}(\tau)$ is an extension of $S(X)$, including Gao's constant $f(X)$ [12] as a special case.

Notation. $J_{t}:=J_{X, t}(1), J:=J(X), S_{t}:=S_{X, t}(1)$ and $S:=S(X)$.

\section{Main results}

3.1. James type constants. The modulus of convexity $\delta_{X}:[0,2] \rightarrow$ $[0,1]$ is defined as

$$
\delta_{X}(\epsilon)=\inf \left\{1-\|x+y\| / 2: x, y \in S_{X},\|x-y\| \geq \epsilon\right\} .
$$

Obviously, $\delta_{X}$ is nondecreasing on $[0,2]$; moreover, the function $\delta_{X}(\epsilon) / \epsilon$ is also nondecreasing on $(0,2]$ (see [11]). The equality

$$
J=\sup _{0 \leq \epsilon \leq 2}\left\{\epsilon: \delta_{X}(\epsilon) \leq 1-\epsilon / 2\right\}
$$

holds for any nontrivial space, while the equality

$$
1-\delta_{X}(J)=J / 2
$$

holds whenever $X$ is uniformly nonsquare (see [8]). 
We recall several known results on the modulus of convexity:

$$
A_{2}(X)=1+\sup _{\sqrt{2} \leq \epsilon \leq 2}\left\{\epsilon / 2-\delta_{X}(\epsilon)\right\}
$$

by Baronti, Casini and Papini [6, Proposition 2.4];

$$
T(X)=\sup _{0 \leq \epsilon \leq 2} \sqrt{2 \epsilon\left(1-\delta_{X}(\epsilon)\right)}
$$

by Alonso and Llorens-Fuster [1, Theorem 11]; and

$$
C_{\mathrm{NJ}}^{\prime}(X)=\sup _{0 \leq \epsilon \leq 2}\left\{\epsilon^{2} / 4+\left(1-\delta_{X}(\epsilon)\right)^{2}\right\}
$$

by Alonso, Martín and Papini [2, Proposition 4]. We generalize the formulas above as follows.

Theorem 3.1. Let $t \in \mathbb{R}$. Then for any Banach space $X$,

$$
J_{t}=\sup \left\{\mathcal{M}_{t}\left(\epsilon, 2\left(1-\delta_{X}(\epsilon)\right)\right): J \leq \epsilon \leq 2\right\} .
$$

Proof. Let $\epsilon \in[0,2]$ and let $\eta>0$ be sufficiently small. Then there exist $x, y \in S_{X}$ with $\|x-y\|=\epsilon$ and $\|x+y\| \geq 2\left(1-\delta_{X}(\epsilon)\right)-\eta$. Therefore

$$
\mathcal{M}_{t}\left(\epsilon, 2\left(1-\delta_{X}(\epsilon)\right)-\eta\right) \leq \mathcal{M}_{t}(\|x-y\|,\|x+y\|) \leq J_{t} .
$$

Since $\epsilon$ is arbitrary, by letting $\eta \rightarrow 0$ we get

$$
J_{t} \geq \sup _{0 \leq \epsilon \leq 2} \mathcal{M}_{t}\left(\epsilon, 2\left(1-\delta_{X}(\epsilon)\right)\right) \geq \sup _{J \leq \epsilon \leq 2} \mathcal{M}_{t}\left(\epsilon, 2\left(1-\delta_{X}(\epsilon)\right)\right) .
$$

To show the opposite inequality let $x, y \in S_{X}$. If $\max (\|x-y\|,\|x+y\|) \geq J$, then we let $\|x-y\|=\epsilon$ and assume without loss of generality that $\|x-y\|=$ $\max (\|x-y\|,\|x+y\|)$. It follows that $\epsilon \geq J,\|x+y\| \leq 2\left(1-\delta_{X}(\epsilon)\right)$ and

$$
\begin{aligned}
\mathcal{M}_{t}(\|x-y\|,\|x+y\|) & \leq \mathcal{M}_{t}\left(\epsilon, 2\left(1-\delta_{X}(\epsilon)\right)\right) \\
& \leq \sup _{J \leq \epsilon \leq 2} \mathcal{M}_{t}\left(\epsilon, 2\left(1-\delta_{X}(\epsilon)\right)\right) .
\end{aligned}
$$

Otherwise, if $\max (\|x-y\|,\|x+y\|) \leq J$, then

$$
\begin{aligned}
\mathcal{M}_{t}(\|x-y\|,\|x+y\|) & \leq J=\mathcal{M}_{t}\left(J, 2\left(1-\delta_{X}(J)\right)\right) \\
& \leq \sup _{J \leq \epsilon \leq 2} \mathcal{M}_{t}\left(\epsilon, 2\left(1-\delta_{X}(\epsilon)\right)\right) .
\end{aligned}
$$

Altogether,

$$
J_{t} \leq \sup _{J \leq \epsilon \leq 2} \mathcal{M}_{t}\left(\epsilon, 2\left(1-\delta_{X}(\epsilon)\right)\right)
$$

which completes the proof.

Remark 3.2. To compute $J_{t}$, it suffices to consider the function $\mathcal{M}_{t}(\epsilon$, $\left.2\left(1-\delta_{X}(\epsilon)\right)\right)$ on $[J, 2]$ instead of $[0,2]$. So our result generalizes and improves some known results. 
EXAmple $3.3\left(\ell_{\infty}-\ell_{1}\right.$ space $)$. Let $X$ be $\mathbb{R}^{2}$ with the norm

$$
\|x\|=\left\|\left(x_{1}, x_{2}\right)\right\|= \begin{cases}\max \left(\left|x_{1}\right|,\left|x_{2}\right|\right) & \text { if } x_{1} x_{2} \geq 0 \\ \left|x_{1}\right|+\left|x_{2}\right| & \text { if } x_{1} x_{2} \leq 0 .\end{cases}
$$

Since $\delta_{X}(\epsilon)=\max (0,(\epsilon-1) / 2)$ and $J=3 / 2$ (see [15, 16]), we get

$$
J_{t}=\max _{3 / 2 \leq \epsilon \leq 2}\left(\frac{\epsilon^{t}+(3-\epsilon)^{t}}{2}\right)^{1 / t} .
$$

It is easy to see that $J_{t}=3 / 2$ for $t \leq 1$ and

$$
J_{t}=\left(\frac{1+2^{t}}{2}\right)^{1 / t} \quad \text { for } t \geq 1
$$

EXAmple $3.4\left(\ell_{2}-\ell_{1}\right.$ space). Let $X$ be $\mathbb{R}^{2}$ with the norm

$$
\|x\|=\left\|\left(x_{1}, x_{2}\right)\right\|= \begin{cases}\left(\left|x_{1}\right|^{2}+\left|x_{2}\right|^{2}\right)^{1 / 2} & \text { if } x_{1} x_{2} \geq 0 \\ \left|x_{1}\right|+\left|x_{2}\right| & \text { if } x_{1} x_{2} \leq 0\end{cases}
$$

Since $J=\sqrt{8 / 3}$ and

$$
\delta_{X}(\epsilon)=1-\sqrt{1-\epsilon^{2} / 8} \quad \text { for } \sqrt{8 / 3} \leq \epsilon \leq 2
$$

(see [15, 16]), we get

$$
J_{t}=\max _{\sqrt{8 / 3} \leq \epsilon \leq 2}\left(\frac{\epsilon^{t}+\left(4-\epsilon^{2} / 2\right)^{t / 2}}{2}\right)^{1 / t} .
$$

A simple calculation shows that

$$
J_{t}=\sqrt{2}\left(\frac{1+2^{t / 2}}{2}\right)^{1 / t} \quad \text { for } t \geq 0,
$$

and

$$
J_{t}=2\left(\frac{1+2^{t /(t-2)}}{2}\right)^{1 / t-1 / 2} \quad \text { for } t \leq 0 .
$$

We now extend and improve inequalities (1.2)-(1.5) to the following form.

Theorem 3.5. Let $t \geq 0$. Then for any Banach space $X$,

$$
J_{t} \leq \frac{2 \mathcal{M}_{t}(J, 2(J-1))}{J} .
$$

Proof. Since in the case $J=2$ the inequality is obvious, we assume $J<2$, that is, $X$ is (UNS). By the monotonicity of $\delta_{X}(\epsilon) / \epsilon$ and (3.1), we have

$$
\delta_{X}(\epsilon) \geq \frac{\delta_{X}(J)}{J} \epsilon=\frac{(2-J) \epsilon}{2 J}=\frac{(S-1) \epsilon}{2}
$$


for any $\epsilon \geq J$. This together with Theorem 3.1 yields

$$
\begin{aligned}
J_{t} & =\sup _{J \leq \epsilon \leq 2} \mathcal{M}_{t}\left(\epsilon, 2\left(1-\delta_{X}(\epsilon)\right)\right) \\
& \leq \sup _{J \leq \epsilon \leq 2} \mathcal{M}_{t}(\epsilon, 2-(S-1) \epsilon)=2 \mathcal{M}_{t}(1,2-S) \\
& =\frac{2 \mathcal{M}_{t}(J, 2(J-1))}{J} .
\end{aligned}
$$

REMARK 3.6. (1) When $X$ is (UNS), by a simple calculation, we can extend the above result from $[0,+\infty)$ to $\left[t_{0},+\infty\right)$, where $t_{0}=1-1 / \log _{S-1}(2-S)$. The upper bound of $J_{t}$ is $\mathcal{M}_{t}\left(\epsilon_{t}, 2-(S-1) \epsilon_{t}\right)$ with $\epsilon_{t}=2 /\left(S-1+(S-1)^{1 /(1-t)}\right)$ whenever $t<t_{0}$.

(2) It is readily seen that inequalities (1.2)-(1.5) are all included in (3.3), which shows that our result generalizes some known results.

Corollary 3.7. $X$ is (UNS) $\Leftrightarrow J_{t}<2$ for some $t \in \mathbb{R}$.

Proof. By the definition of $J_{t}$, it is not hard to check that $J_{t} \leq 2$ for all $t \in \mathbb{R}$. Thus our assertion is equivalent to $J=2 \Leftrightarrow J_{t}=2$ for all $t \in \mathbb{R}$. That $J=2 \Rightarrow J_{t}=2$ for all $t \in \mathbb{R}$ follows from $J_{t} \geq J$. To see the converse, let $t \geq 0$ be fixed. By assumption $J_{t}=2$. It follows from (3.3) that

$$
J=J\left(J_{t} / 2\right) \leq \mathcal{M}_{t}(J, 2(J-1)) \leq J,
$$

which implies $\mathcal{M}_{t}(J, 2(J-1))=J$ and thus $J=2$.

3.2. Schäffer type constant. The modulus of smoothness $\varrho_{X}:[0,2] \rightarrow$ $[0,1]$ is defined as

$$
\varrho_{X}(\epsilon)=\sup \left\{1-\|x+y\| / 2: x, y \in S_{X},\|x-y\| \leq \epsilon\right\} .
$$

It is known that $\varrho_{X}$ is continuous and convex on $[0,2]$; moreover, the function $\varrho_{X}(\epsilon) / \epsilon$ is also nondecreasing on $(0,2]$. In addition

$$
1-\varrho_{X}(S)=S / 2
$$

for any Banach space $X$ (see e.g. [3, 4, 7, 5]). By using related properties, we state a relation between this modulus and Schäffer type constants.

Theorem 3.8. Let $t>1$. Then for any Banach space $X$,

$$
S_{t}=\min \left\{\mathcal{M}_{t}\left(\epsilon, 2\left(1-\varrho_{X}(\epsilon)\right)\right): 0 \leq \epsilon \leq S\right\} .
$$

Proof. Let $\epsilon \in[0,2]$ be fixed. For sufficiently small $\eta>0$, there exist $x, y \in S_{X}$ with $\|x-y\|=\epsilon$ so that $\|x+y\| \leq 2\left(1-\varrho_{X}(\epsilon)\right)+\eta$. Hence

$$
\mathcal{M}_{t}\left(\epsilon, 2\left(1-\varrho_{X}(\epsilon)\right)+\eta\right) \geq \mathcal{M}_{t}(\|x-y\|,\|x+y\|) \geq S_{t} .
$$

Since $\epsilon$ is arbitrary, by letting $\eta \rightarrow 0$ we obtain

$$
S_{t} \leq \inf _{0 \leq \epsilon \leq 2} \mathcal{M}_{t}\left(\epsilon, 2\left(1-\varrho_{X}(\epsilon)\right)\right) \leq \inf _{0 \leq \epsilon \leq S} \mathcal{M}_{t}\left(\epsilon, 2\left(1-\varrho_{X}(\epsilon)\right)\right) .
$$


To see the opposite inequality, let $x, y \in S_{X}$. If $\min (\|x-y\|,\|x+y\|) \leq S$, then we assume without loss of generality that $\|x-y\|=\min (\|x-y\|,\|x+y\|)$ and let $\|x-y\|=\epsilon$. Then $\epsilon \leq S,\|x+y\| \geq 2\left(1-\varrho_{X}(\epsilon)\right)$ and

$$
\mathcal{M}_{t}(\|x-y\|,\|x+y\|) \geq \mathcal{M}_{t}\left(\epsilon, 2\left(1-\varrho_{X}(\epsilon)\right)\right) \geq \inf _{0 \leq \epsilon \leq S} \mathcal{M}_{t}\left(\epsilon, 2\left(1-\varrho_{X}(\epsilon)\right)\right) .
$$

Otherwise, if $\min (\|x-y\|,\|x+y\|) \geq S$, then

$$
\begin{aligned}
\mathcal{M}_{t}(\|x-y\|,\|x+y\|) & \geq S=\mathcal{M}_{t}\left(S, 2\left(1-\varrho_{X}(S)\right)\right) \\
& \geq \inf _{0 \leq \epsilon \leq S} \mathcal{M}_{t}\left(\epsilon, 2\left(1-\varrho_{X}(\epsilon)\right)\right) .
\end{aligned}
$$

Altogether

$$
S_{t} \geq \inf _{0 \leq \epsilon \leq S} \mathcal{M}_{t}\left(\epsilon, 2\left(1-\varrho_{X}(\epsilon)\right)\right) .
$$

Thus the result follows from the continuity of $\varrho_{X}(\epsilon)$.

Example 3.9. Let $X$ be the $\ell_{\infty} \ell_{1}$ space defined in Example 3.3. From $\varrho_{X}(\epsilon)=\max (\epsilon / 4, \epsilon-1)$ (see [7]), it follows that

$$
S_{t}=\min _{0 \leq \epsilon \leq 4 / 3}\left(\frac{\epsilon^{t}+(2-\epsilon / 2)^{t}}{2}\right)^{1 / t}=2\left(\frac{2}{1+2^{t^{\prime}}}\right)^{1 / t^{\prime}},
$$

where $t^{\prime}>1,1 / t+1 / t^{\prime}=1$.

EXAmPle $3.10\left(\ell_{2}-\ell_{\infty}\right.$ space). Let $X$ be $\mathbb{R}^{2}$ with the norm

$$
\|x\|=\left\|\left(x_{1}, x_{2}\right)\right\|= \begin{cases}\left(\left|x_{1}\right|^{2}+\left|x_{2}\right|^{2}\right)^{1 / 2} & \text { if } x_{1} x_{2} \geq 0 \\ \max \left(\left|x_{1}\right|,\left|x_{2}\right|\right) & \text { if } x_{1} x_{2} \leq 0 .\end{cases}
$$

Since $J=1+1 / \sqrt{2}$, we have $S=2(2-\sqrt{2})$. It is proved in [10] that

$$
\varrho_{X}(\epsilon)=\max \left(\frac{\epsilon}{2 \sqrt{2}}, \frac{\epsilon}{\sqrt{2}}+1-\sqrt{2}\right)
$$

which implies that

$$
S_{t}=\min _{0 \leq \epsilon \leq 2(2-\sqrt{2})}\left(\frac{\epsilon^{t}+(2-\epsilon / \sqrt{2})^{t}}{2}\right)^{1 / t}=\sqrt{2}\left(\frac{2}{1+2^{t^{\prime} / 2}}\right)^{1 / t^{\prime}},
$$

where $t^{\prime}>1,1 / t+1 / t^{\prime}=1$.

It is readily seen that $S_{t} \leq S$ for every $t>1$. By the formula $J S=2$, we see that the upper bound of $S_{t}$ is $2 / J$. We now consider its lower bound.

Theorem 3.11. For any Banach space $X$,

$$
S_{t} \geq 1 / \mathcal{M}_{t^{\prime}}(1, J-1),
$$

where $t, t^{\prime}>1$ with $1 / t+1 / t^{\prime}=1$. 
Proof. Since $\varrho_{X}(\epsilon) / \epsilon$ is nondecreasing and $2\left(1-\varrho_{X}(S)\right)=S$, it follows that

$$
\varrho_{X}(\epsilon) \leq \frac{\varrho_{X}(S)}{S} \epsilon=\frac{(2-S) \epsilon}{2 S}=\frac{(J-1) \epsilon}{2}
$$

for all $\epsilon \in[0, S]$. Using Theorem 3.8 now yields

$$
S_{t}=\min _{0 \leq \epsilon \leq S} \mathcal{M}_{t}\left(\epsilon, 2\left(1-\varrho_{X}(\epsilon)\right)\right) \geq \min _{0 \leq \epsilon \leq S} \mathcal{M}_{t}(\epsilon, 2-(J-1) \epsilon) .
$$

Since $\mathcal{M}_{t}(\epsilon, 2-(J-1) \epsilon)$ attains its minimum at

$$
\epsilon_{t}:=\frac{2}{(J-1)+(J-1)^{-1 /(t-1)}} \leq \frac{2}{(J-1)+1}=S,
$$

we infer that

$$
S_{t} \geq \mathcal{M}_{t}\left(\epsilon_{t}, 2-(J-1) \epsilon_{t}\right)=1 / \mathcal{M}_{t^{\prime}}(1, J-1) .
$$

REMARK 3.12. (1) Example 3.9 shows that inequality (3.4) is sharp even for a uniformly nonsquare space.

(2) Theorem 3.11 is an improvement of [23, Theorem 2]. In fact, for $t=2$, inequality (3.4) is reduced to

$$
f(X) \geq \frac{4}{1+(J-1)^{2}},
$$

where $f(X)=2\left(S_{2}\right)^{2}$ is a constant introduced in [12].

Corollary 3.13. $X$ is $(U N S) \Leftrightarrow S_{t}>1$ for some $t>1$.

Proof. By the definition of $S_{t}$, we know that $S_{t} \geq 1$ for all $t>1$. Thus our assertion is equivalent to $J=2 \Leftrightarrow S_{t}=1$ for all $t>1$. That $J=2 \Rightarrow S_{t}=1$ for all $t>1$ follows from the estimate $S_{t} \leq 2 / J$. To see the converse, we deduce from (3.4) that

$$
1 \geq \mathcal{M}_{t^{\prime}}(1, J-1) \geq 1 / S_{t}=1,
$$

which implies $\mathcal{M}_{t^{\prime}}(1, J-1)=1$ and thus $J=2$.

3.3. Zbăganu constant. The Zbăganu constant, introduced in [27], is defined as

$$
C_{\mathrm{Z}}(X)=\sup \left\{\frac{\|x+y\|\|x-y\|}{\|x\|^{2}+\|y\|^{2}}: x \in S_{X}, y \in B_{X}\right\} .
$$

Recently Takahashi studied the relation between the Zbăganu and James constants and proved the following inequality (see [19, Theorem 18]):

$$
C_{\mathrm{Z}}(X) \leq \frac{J+\sqrt{J^{2}+(2-J)^{2}}}{2}
$$

Applying the previous result, we can improve (3.5) to the following form. 
Theorem 3.14. For any Banach space $X$,

$$
C_{\mathrm{Z}}(X) \leq \frac{2(J-1)+\sqrt{4(J-1)^{2}+(2-J)^{2}}}{J} .
$$

Proof. Observe first that $\|x \pm \tau y\| \leq \tau\|x \pm y\|+(1-\tau)$ for every $0 \leq \tau \leq 1$ and $x \in S_{X}$. This together with Theorem 3.5 leads to

$$
\begin{aligned}
\frac{J_{X, 0}^{2}(\tau)}{1+\tau^{2}} & =\sup \left\{\frac{\|x+\tau y\|\|x-\tau y\|}{1+\tau^{2}}: x, y \in S_{X}\right\} \\
& \leq \frac{J_{X, 0}^{2}(1) \tau^{2}+2 J_{X, 1}(1) \tau(1-\tau)+(1-\tau)^{2}}{1+\tau^{2}} \\
& \leq \frac{(3 J-4) \tau^{2}+4(J-1) \tau+J}{J\left(1+\tau^{2}\right)} .
\end{aligned}
$$

It follows from a simple computation that

$$
\begin{aligned}
C_{\mathrm{Z}}(X) & =\sup \left\{\frac{J_{X, 0}^{2}(\tau)}{1+\tau^{2}}: 0 \leq \tau \leq 1\right\} \\
& \leq \max _{0 \leq \tau \leq 1} \frac{(3 J-4) \tau^{2}+4(J-1) \tau+J}{J\left(1+\tau^{2}\right)} \\
& =\frac{2(J-1)+\sqrt{4(J-1)^{2}+(2-J)^{2}}}{J} .
\end{aligned}
$$

REMARK 3.15. Since $\sqrt{2} \leq J \leq 2$, one gets

$$
\frac{2(J-1)+\sqrt{4(J-1)^{2}+(2-J)^{2}}}{J} \leq J \leq \frac{J+\sqrt{J^{2}+(2-J)^{2}}}{2} .
$$

Indeed, the right-hand inequality is obvious and the left-hand inequality follows from the following chain:

$$
\begin{aligned}
& 2(J-1)+\sqrt{4(J-1)^{2}+(2-J)^{2}} \leq J^{2} \\
& \Leftrightarrow 4(J-1)^{2}+(2-J)^{2} \leq\left(1+(J-1)^{2}\right)^{2} \\
& \Leftrightarrow(2-J)^{2} \leq\left(1-(J-1)^{2}\right)^{2} \\
& \Leftrightarrow(J-1)^{2} \leq J-1 .
\end{aligned}
$$

Thus our result significantly improves (3.5).

Acknowledgements. We would like to express our sincere appreciation to the referee for his/her valuable suggestions and for pointing out an error in the proof of Theorem 3.5 .

The second author is supported by the Technology and Pioneering project in Henan province (102300410012). 


\section{References}

[1] J. Alonso and E. Llorens-Fuster, Geometric mean and triangles inscribed in a semicircle in Banach spaces, J. Math. Anal. Appl. 340 (2008), 1271-1283.

[2] J. Alonso, P. Martín and P. L. Papini, Wheeling around von Neumann-Jordan constant in Banach spaces, Studia Math. 188 (2008), 135-150.

[3] J. Banaś, On modulus of smoothness of Banach spaces, Bull. Polish Acad. Sci. Math. 34 (1986), 287-293.

[4] J. Banaś and K. Frączek, Deformation of Banach spaces, Comment. Math. Univ. Carolin. 34 (1993), 47-53.

[5] J. Banaś and B. Rzepka, Functions related to convexity and smoothness of normed spaces, Rend. Circ. Mat. Palermo (2) 46 (1997), 395-424.

[6] M. Baronti, E. Casini and P. L. Papini, Triangles inscribed in a semicircle, in Minkowski planes, and in normed spaces, J. Math. Anal. Appl. 252 (2000), 124-146.

[7] M. Baronti and P. L. Papini, Convexity, smoothness and moduli, Nonlinear Anal. 70 (2009), 2457-2465.

[8] E. Casini, About some parameters of normed linear spaces, Atti Accad. Naz. Lincei Rend. Cl. Sci. Fis. Mat. Natur. 80 (1986), 11-15.

[9] J. A. Clarkson, The von Neumann-Jordan constant for the Lebesgue spaces, Ann. of Math. 38 (1937), 114-115.

[10] H. H. Cui and Y. R. Zhang, A note on the Banaś modulus of smoothness in the Bynum space, Appl. Math. Lett. 23 (2010), 299-301.

[11] T. Figiel, On the moduli of convexity and smoothness, Studia Math. 56 (1976), 121-155.

[12] J. Gao, A Pythagorean approach in Banach spaces, J. Inequal. Appl. art. ID 94982, (2006), 1-11.

[13] J. Gao and K. S. Lau, On the geometry of spheres in normed linear spaces, J. Austral. Math. Soc. Ser. A 48 (1990), 101-112.

[14] - - - On two classes of Banach spaces with uniform normal structure, Studia Math. 99 (1991), 41-56.

[15] K. Goebel and W. A. Kirk, Topics in Metric Fixed Point Theory, Cambridge Univ. Press, 1990.

[16] M. Kato, L. Maligranda and Y. Takahashi, On James and Jordan-von Neumann constants and the normal structure coefficient of Banach spaces, Studia Math. 144 (2001), 275-295.

[17] L. Maligranda, L. I. Nikolova, L. E. Persson and T. Zachariades, On n-th James and Khintchine constants of Banach spaces, Math. Inequal. Appl. 11 (2008), 1-22.

[18] S. Saejung, On James and von Neumann-Jordan constants and sufficient conditions for the fixed point property, J. Math. Anal. Appl. 323 (2006), 1018-1024.

[19] Y. Takahashi, Some geometric constants of Banach spaces - A unified approach, in: Banach and Function Spaces II, Yokohama Publ., 2006, 191-220.

[20] Y. Takahashi and M. Kato, A simple inequality for the von Neumann-Jordan and James constants of a Banach space, J. Math. Anal. Appl. 359 (2009), 602-609.

[21] F. H. Wang, On the James and von Neumann-Jordan constants in Banach spaces, Proc. Amer. Math. Soc. 138 (2010), 695-701.

[22] F. H. Wang and B. J. Pang, Some inequalities concerning the James constant in Banach spaces, J. Math. Anal. Appl. 353 (2009), 305-310.

[23] F. H. Wang and C. S. Yang, Uniform nonsquareness, uniform normal structure and Gao's constants, Math. Inequal. Appl. 11 (2008), 607-614.

[24] C. S. Yang, A note on Jordan-von Neumann constant and James constant, J. Math. Anal. Appl. 357 (2009), 98-102. 
[25] C. S. Yang and H. Li, An inequality between Jordan-von Neumann constant and James constant, Appl. Math. Lett. 23 (2010), 277-281.

[26] C. S. Yang and F. H. Wang, On a new geometric constant related to the von Neumann-Jordan constant, J. Math. Anal. Appl. 324 (2006), 555-565.

[27] G. Zbăganu, An inequality of $M$. Rădulescu and S. Rădulescu which characterizes the inner product spaces, Rev. Roumaine Math. Pures Appl. 47 (2002), 253-258.

Fenghui Wang

Department of Mathematics

Luoyang Normal University

Luoyang 471022, China

E-mail: wfenghui@gmail.com
Changsen Yang

Department of Mathematics

Henan Normal University

Xinxiang 453007, China

E-mail: yangchangsen0991@sina.com

Received March 17, 2010

Revised version September 23, 2010 